\title{
PrEP Awareness and Perceived Barriers Among Single Young Men who have Sex with Men
}

\author{
Jose A. Bauermeister*, Steven Meanley, Emily Pingel, Jorge H. Soler and Gary W. Harper
}

The Center for Sexuality \& Health Disparities, School of Public Health, University of Michigan, Ann Arbor, MI, USA

\begin{abstract}
Pre-exposure prophylaxis (PrEP) has the potential to help reduce new HIV infections among young men who have sex with men (YMSM). Using a cross-sectional survey of YMSM (N=1,507; ages 18-24), we gauged YMSM's PrEP awareness and PrEP-related beliefs regarding side effects, accessibility, and affordability. Overall, 27\% of the sample had heard about PrEP; $1 \%$ reported ever using PrEP prior to sex. In a multivariate logistic regression, we found that YMSM were more likely to have heard about PrEP if they were older, more educated, were residentially unstable in the prior 30 days, had insurance, or reported having at least one sexually transmitted infection in their lifetime. We found no differences by race/ethnicity, history of incarceration, or recent sexual risk behavior. In multivariate linear regression models, Black and Latino YMSM were more likely than Whites to state they would not use PrEP because of side effect concerns. YMSM were more likely to indicate that they would not be able to afford PrEP if they did not have insurance or if they had a prior sexually transmitted infection, PrEP rollout may be hindered due to lack of awareness, as well as perceived barriers regarding its use. We propose strategies to maximize equity in PrEP awareness and access if it is to be scaled up among YMSM.
\end{abstract}

Keywords: Access, barriers, biomedical, HIV, MSM, prevention.

\section{INTRODUCTION}

Pre-Exposure Prophylaxis (PrEP) involves the use of antiretroviral medications (e.g., tenofovir and emtricitabine) prior to potential exposure to HIV. In a multi-national trial (iPrEx), the efficacy of daily oral tenofovir and emtricitabine use was tested among 3,000 men who have sex with men (MSM) in six countries [1]. There were $44 \%$ fewer HIV infections among participants receiving the oral PrEP combination alongside a comprehensive HIV psychosocial prevention package (e.g., regular HIV testing, access to health care for toxicity evaluations and/or treatment for any HIV-related complications). These results led the US Food and Drug Administration to approve the use of Truvada, a combination of tenofovir and emtricitabine, as a PrEP treatment for MSM in 2012. Although behavioral researchers have documented willingness to use PrEP among MSM populations disproportionately affected by HIV/AIDS, including African Americans [2] and individuals of lower socioeconomic status [3], the implementation of PrEP will require us to address barriers associated with PrEP awareness, side effects, access, and affordability in these communities [4].

The combination of PrEP and psychosocial HIV prevention measures could help curtail the incidence of HIV/AIDS among young men who have sex with men (YMSM). More than half of all new HIV infections are transmitted through sexual contact among MSM [5]. In 2009, YMSM accounted for $44 \%$ of all MSM infections, $27 \%$ of new infections nationwide, and close to $70 \%$ of new

*Address correspondence to this author at the University of Michigan School of Public Health, 1415 Washington Heights, SPH I, Room 3822, Ann Arbor, MI 48109-2029, USA; Tel: (734)764-6653; Fax: (734)763-7259; E-mail: jbauerme@umich.edu infections among individuals aged 13-29 [6]. African American and Latino YMSM, in particular, accounted for the largest proportion of new HIV infections among MSM in this age group [7]. These racial/ethnic disparities have been linked to sociodemographic characteristics such as educational attainment and income $[8,9]$, as well as structural vulnerabilities including residential instability and homelessness [10], lack of affordable access to comprehensive health services [11], and a history of incarceration [12]. These social vulnerabilities warrant further scrutiny, as they may also affect YMSM's awareness of and/or willingness to use PrEP. Consequently, as a contribution to this literature, we sought to examine YMSM's concerns regarding PrEP-related side effects, access, and affordability.

Given that a small proportion of iPrEx trial participants were YMSM, it remains vital that we gauge YMSM's awareness of PrEP and address their perceived barriers regarding side effects, access, and affordability, as we develop YMSM-specific PrEP interventions [13]. From a theoretical standpoint, PrEP-related interventions will have to address YMSM's perceived psychosocial barriers regarding PrEP, as this construct has been documented to be one of the strongest predictors of behavior change and maintenance $[14,15]$. In a recent qualitative study, for example, Smith and colleagues [16] noted that African American YMSM's interest in PrEP was contingent upon its perceived cost and accessibility, as well as their ability to access health care. Similarly, Mustanski and colleagues [17] found that PrEP interest among YMSM was associated with perceptions of low side effect burden. Although these findings parallel prior findings with adult MSM [3], PrEPrelated concerns are particularly salient in PrEP implementation for YMSM as they are less likely to have access to routine and quality medical care $[18,19]$, may be more vulnerable to PrEP-related side effects such as adverse bone effects [20], and may not be able to afford PrEP 
medication and its associated toxicology screenings. Given these concerns and the need to develop developmentally and culturally appropriate intervention strategies to address these barriers, we sought to examine YMSM's perceived barriers regarding PrEP accessibility, affordability, and side effects.

Researchers have also cautioned that although PrEP may offer an alternative prevention strategy to MSM who find condom use difficult, it could also promote risk disinhibition by encouraging lack of condom use [21]. Although researchers have found no evidence of risk disinhibition in clinical trials, the absence of risk disinhibition may be attributable to the comprehensive psychosocial services offered as part of the trials (e.g., access to behavioral counseling, free condoms, treatment for sexually transmitted infections [STIs], regular HIV testing, and frequent medical evaluation) and limited number of youth recruited. In nonclinical behavioral studies, researchers have found mixed results regarding PrEP willingness and condom use disinhibition. Using a sample of HIV-negative MSM living in New York City, Golub et al. [2] found that over 35\% of MSM who would use PrEP also indicated they would be less likely to use condoms while using PrEP. In a recent study with YMSM (ages 16-20) in Chicago, however, Mustanski and colleagues [17] found PrEP intentions were associated with fewer unprotected anal sex acts. Consequently, it remains unclear whether YMSM's health promotion and risk practices may influence their PrEP intentions, and/or whether these findings are generalizable to other geographic areas. We build upon this body of work by examining whether PrEP awareness or perceived barriers regarding side effects, access, and affordability were associated with recent sexual risk (i.e., unprotected receptive anal intercourse) and prior HIV/STI infections in a sample of YMSM recruited across the United States.

\section{Study Goals and Objectives}

The goal of the current study was to examine PrEP awareness and perceived barriers among single YMSM, ages 18-24, in the United States. We focus on single men, as they may benefit from PrEP if they decide to forego condoms with new casual and romantic partners. Given the limited data focused solely on YMSM, we first examined the prevalence of PrEP awareness in our sample and compared it across sociodemographic variables (e.g., age, race/ethnicity, education), structural vulnerabilities (e.g., residential instability, history of incarceration, lack of insurance coverage), and sexual risk outcomes (e.g., recent unprotected receptive anal intercourse, a history of STIs). Among those who reported being aware of PrEP, we then examined whether their PrEP-related perceived barriers regarding side effects, accessibility, and affordability, respectively, varied across these sociodemographic, structural, and sexual risk correlates.

\section{METHODS}

\section{Sample}

Data for this paper come from a cross-sectional observational study examining single YMSM's partner- seeking experiences online between July 2012 and January 2013. To be eligible for participation, recruits had to selfidentify as male, be between the ages of 18 and 24, selfidentify as single, report having ever gone online to meet a male partner for dating and/or sex, and be a resident of the United States (including Puerto Rico).

A total of 3,140 entries were recorded over 7 months of data collection. We excluded 942 entries because they were ineligible to participate once they completed the screener. We then used best practices [22] to identify duplicates and falsified entries by manually examining participants' online presence, email and IP addresses, operating system and browser information, irregular answer patterns, and time taken to complete survey. We disqualified 366 entries because they were identified as duplicate/ fraudulent entries, leaving us with a total of 1,963 valid entries. Of these, 325 participants consented but did not commence the survey (i.e., missing all data; $16.6 \%$ ); resulting in an analytic sample of $N$ $=1,638$ eligible YMSM. One hundred and ninety-three of these eligible and consented participants did not complete all sections of the survey (i.e., missing data in some sections of the survey; $10.5 \%$ ). For those questionnaires that were incomplete, participants were sent two reminder emails that encouraged them to complete the questionnaire; one email was sent a week after they had started the questionnaire and another was sent a week before the questionnaire was scheduled to close.

For the purposes of this analysis, we report on the subsample that provided full study data $(N=1,507 ; 76.8 \%$ participation rate). The sample had a mean age of 20.8 years $(\mathrm{SD}=1.92)$. The racial/ethnic distribution of our sample was predominantly White/Caucasian $(\mathrm{N}=987 ; 65.5 \%)$, followed by Latino/Hispanic $(\mathrm{N}=256 ; 17.0 \%)$, Black/African American $(\mathrm{N}=131 ; 8.7 \%)$, Asian/Pacific Islander $(\mathrm{N}=58$; $3.8 \%)$, Multiracial $(\mathrm{N}=54 ; 3.6 \%)$ or other $(\mathrm{N}=21 ; 1.4 \%)$. Participants who completed our survey lived in 49 of the 50 US states and territories, including Puerto Rico and Washington D.C. (18.8\% from the Northeast, $26.1 \%$ from the Midwest, $27.9 \%$ from the South, $26.0 \%$ from the West, and $0.8 \%$ from Puerto Rico; 6 participants did not provide information on their state of residence). We provide a brief description of the sample's characteristics in Table $\mathbf{1 .}$

\section{Procedures}

Participants were primarily recruited through advertisements on two popular social networking sites and participant referrals. Social network advertisements were viewable only to men who fit our age range and who lived in the United States. Promotional materials displayed a synopsis of eligibility criteria, a mention of a \$10 VISA egift card incentive, and the survey's website. In our advertisements, we did not define "dating websites" so as to ensure inclusivity of sites that are marketed for romance or for broader social purposes such as "hooking up". Sites typically used for "hooking up" or "seeking casual sex" were included under the assumption that some youth seek casual sex on the path to finding romantic partners. By using these sites, we could account for participants who may be using them for purposes beyond casual sex. 
Table 1. Demographic Characteristics of Study Sample $(\mathbf{N}=$ 1507)

\begin{tabular}{|c|c|}
\hline & Mean (SD)/N (\%) \\
\hline Age & $20.80(.12)$ \\
\hline \multicolumn{2}{|l|}{ Race/Ethnicity } \\
\hline White & $987(65.5 \%)$ \\
\hline Black & $131(8.7 \%)$ \\
\hline Latino & $256(17.0 \%)$ \\
\hline Asian/Pacific Islander & $58(3.8 \%)$ \\
\hline Multiracial & $54(3.6 \%)$ \\
\hline Other & $21(1.4 \%)$ \\
\hline \multicolumn{2}{|l|}{ Sexual Identity } \\
\hline Gay & $1389(92.2 \%)$ \\
\hline Bisexual & $45(3.2 \%)$ \\
\hline Other & $68(4.6 \%)$ \\
\hline \multicolumn{2}{|l|}{ Educational Attainment } \\
\hline Less than high school degree & $54(3.6 \%)$ \\
\hline High school degree & $318(21.1 \%)$ \\
\hline Technical/Associate Degree & $89(5.9 \%)$ \\
\hline Some College & $733(48.6 \%)$ \\
\hline College & $220(14.6 \%)$ \\
\hline Some graduate school & $93(6.2 \%)$ \\
\hline Residentially Unstable (30 days) & $210(13.9 \%)$ \\
\hline Ever sent to jail/prison & $113(7.5 \%)$ \\
\hline Covered by insurance & $1221(81.0 \%)$ \\
\hline \multicolumn{2}{|l|}{ Sexual Behaviors } \\
\hline Sexually Active in prior 2 months & $1062(70.5 \%)$ \\
\hline URAI in past 2 months & $427(28.3 \%)$ \\
\hline \multicolumn{2}{|l|}{ Prior HIV/STI } \\
\hline HIV-positive & $19(1.3 \%)$ \\
\hline Other Sexually-Transmitted Infection & $224(14.8 \%)$ \\
\hline Prior PrEP awareness & $410(27.2 \%)$ \\
\hline \multicolumn{2}{|l|}{ PrEP-related beliefs $\mathrm{s}^{\mathrm{a}, \mathrm{b}}$} \\
\hline Side-Effects & $2.30(.93)$ \\
\hline Accessibility & $2.48(1.06)$ \\
\hline Affordability & $2.78(.92)$ \\
\hline
\end{tabular}

${ }^{\mathrm{a}} \mathrm{S}$ cores are computed only for YMSM who reported prior PrEP awareness ( $\left.\mathrm{N}=410\right)$; ${ }^{\mathrm{b}}$ Items scored on a 1-4 scale ( $1=$ Strongly Disagree; $4=$ Strongly Agree).

We developed our web survey using best practices [23], including various iterations of pilot testing prior to data collection. Study data were protected with a 128-bit SSL encryption and kept within a University of Michigan firewalled server. Upon entering the study site, participants were asked to enter a valid and private email address, which served as their username. This allowed participants to save their answers and, if unable to complete the questionnaire in one sitting, continue the questionnaire at a later time. Upon completing an eligibility screener, eligible youth were presented with a detailed consent form that explained the purpose of the study and their rights as participants, and were asked to acknowledge that they had read and understood each section of the consent form. We acquired a Certificate of Confidentiality from the National Institutes of Health to protect study data. The University of Michigan Institutional Review Board approved all study procedures.

Consented participants then answered a 30-45 minute questionnaire that covered assessments regarding their sociodemographic characteristics, HIV status, Internet use, ideal relationship and partner characteristics, sexual and substance use behaviors, general mood over the last few months, and PrEP and microbicide-related awareness and perceived barriers. Participants were compensated with a \$10 VISA egift card via e-mail if they completed the survey or if they had started answering the survey but had incomplete entries by the end of the data collection period.

\section{Measures}

PrEP awareness and PrEP-related perceived barriers: We introduced the section examining PrEP awareness and beliefs with a brief statement summarizing the recent iPrEx findings: "Currently, researchers are examining a medical HIV prevention called Pre-Exposure Prophylaxis, or PrEP. People who use PrEP might have to take a pill either every day or at least a couple of hours prior to sex to counteract or at least decrease the chances of becoming infected if they come in contact with HIV." After reading this statement, we asked participants to note whether they had previously heard about PrEP $(0=$ No, $1=$ Yes $)$. Participants who had heard about PrEP were then asked to identify how they had heard about it: a friend, the media, a medical provider, a sex partner, online, or other. These participants were also asked if they had ever taken PrEP prior to having sex $(0=\mathrm{No}, 1=\mathrm{Yes})$, and whether they had any friends who had taken PrEP prior to sex as a prevention strategy $(0=$ No, $1=$ Yes, $2=$ Not Sure $)$.

Participants were also asked to rate their agreement with three PrEP-related perceived barriers. These three items ascertained their agreement with the following statements: "I would not take PrEP drugs because I am concerned about their side effects", "I would know how to get PrEP drugs if I wanted them" (reverse coded) and "I could not afford PrEP drugs". Participants could answer each question using a 4point scale ( 1 =Strongly Disagree, $4=$ Strongly Agree).

Sociodemographic characteristics: Respondents were asked to report their age, educational attainment, and racial/ethnic group membership. We measured race using the following categories: White/Caucasian, Black/African American, Asian/Pacific Islander, Middle Eastern, Native American, and Other. Participants who selected more than one race (e.g., White/Caucasian and Black/African American) were grouped in a Multi-Racial category. We combined the Middle Eastern, Native American and Other Race categories given the limited number of observations in each. We then created dummy variables for each race/ethnicity group, having White/Caucasian participants serve as the referent group. For ethnicity, respondents were asked to report if they considered themselves Latino or 
Hispanic. Non-Hispanic/Latino participants serve as the referent group.

Structural Vulnerabilities: We also ascertained whether participants reported having spent at least one night in the prior 30 days in any of the following venues: a homeless shelter $(N=6)$, public space not intended for sleeping (e.g., bus station, car, abandoned building) $(N=34)$, on the street $(N=18)$, temporarily doubled up with a friend or family member $(N=183)$, in a temporary housing program $(N=12)$, or in a welfare or voucher hotel/motel $(N=10)$. Participants who experienced any of these situations were categorized as residentially unstable. We created a dummy variable to account for YMSM's residential instability, with no experiences of residential instability in the prior 30 days serving as the referent group $(0=\mathrm{No}, 1=\mathrm{Yes})$. Participants also indicated whether they were covered by any health insurance $(0=$ No, $1=$ Yes $)$, and whether they had ever spent time in jail/prison $(0=\mathrm{No}, 1=\mathrm{Yes})$.

Sexual Behavior: Respondents were asked to report their sexual behavior with men and women during the previous two months using a previously validated assessment for YMSM [24]. Questions were posed both in formal language and vernacular (in italics) to increase comprehension. In Table 1, we report the proportion of participants who reported being sexually active, as defined by having had at least one male sexual partner in the past two months, and whether they reported at least one occasion of unprotected receptive anal intercourse (URAI) over the same period $(0=\mathrm{No}, 1=\mathrm{Yes})$.

HIV and STI diagnoses: Participants were asked whether they had ever been diagnosed with HIV or another STI by a health professional. Upon inspection of these data, we collapsed the small proportion reporting testing HIV-positive $(N=19)$ with those who reported another STI $(N=224)$, and created a dummy variable to identify participants who reported a prior HIV/STI infection. Participants without a diagnosed STI served as referent group $(0=\mathrm{No}, 1=\mathrm{Yes})$. In sensitivity analyses, the observed relationships did not change when we excluded HIVpositive participants from our sample.

\section{Data Analytic Strategy}

We first examined the study variables using descriptive statistics (see Table 1). We then performed a logistic regression to assess whether PrEP awareness was associated with sociodemographic characteristics, prior HIV/STI diagnosis, and having engaged in URAI in the prior two months (see Table 2). Subsequently, we selected the subsample $(\mathrm{N}=410)$ reporting prior PrEP awareness and used multivariate linear regression to examine the association between YMSM's PrEP-related beliefs and their sociodemographic characteristics, prior HIV/STI diagnosis, and having engaged in URAI in the prior two months (see Table 3).

\section{RESULTS}

\section{PrEP Awareness}

Over a quarter of the sample $(N=410 ; 27.2 \%)$ had heard about PrEP prior to completing the survey. These participants reported learning about PrEP through the media
( $N=207,50.5 \%)$, the Internet $(N=157,38.3 \%)$, a friend $(N=64,15.6 \%)$, a medical provider $(N=45,11.0 \%)$, a sex partner $(N=16,3.9 \%)$, and/or through other sources (e.g., class discussion; $N=50,12.2 \%)$. Only a small number of PrEP-aware participants reported having taken PrEP as protection against HIV $(N=11 ; 2.7 \%)$, and having friends who had taken PrEP to avoid becoming HIV infected $(N=15$; $3.7 \%$ ).

When we examined PrEP awareness using a multivariate logistic regression model (see Table 2), we found that YMSM were more likely to have heard about PrEP if they were older $(\mathrm{OR}=1.13 ; p<.001)$, more educated $(\mathrm{OR}=1.19$; $p<.01)$, had insurance $(\mathrm{OR}=1.50 ; p<.05)$, and reported at least one sexually transmitted infection (STI) in their lifetime $(\mathrm{OR}=1.81 ; p<.001)$. Conversely, we found no association between PrEP awareness and race/ethnicity, residential instability, incarceration, or having engaged in URAI in the prior 2 months.

Table 2. Logistic Regression Examining the Relationship of PrEP Awareness and YMSM's Sociodemographic Characteristics

\begin{tabular}{|c|c|c|c|}
\hline & OR & $95 \%$ CI & Sig. \\
\hline Age & 1.13 & $(1.05,1.21)$ & .001 \\
\hline \multicolumn{4}{|l|}{ Race/Ethnicity } \\
\hline Black & 1.09 & $(.71,1.68)$ & .696 \\
\hline Latino & .93 & $(.67,1.29)$ & 660 \\
\hline Asian/Pacific Islander & 1.04 & $(.57,1.89)$ & .908 \\
\hline Multiracial & 1.02 & $(.54,1.93)$ & .964 \\
\hline Other & .68 & $(.22,2.09)$ & .497 \\
\hline Educational Attainment & 1.19 & $(1.07,1.34)$ & .002 \\
\hline Residentially Unstable (30 days) & 1.37 & $(.98,1.91)$ & .069 \\
\hline Ever sent to jail/prison & .63 & $(.38,1.03)$ & .067 \\
\hline Covered by insurance & 1.50 & $(1.07,2.09)$ & .018 \\
\hline URAI in past 2 months & 1.20 & $(.93,1.57)$ & .164 \\
\hline Prior STI/HIV & 1.79 & $(1.31,2.43)$ & .001 \\
\hline (Constant) & .01 & & .001 \\
\hline
\end{tabular}

\section{PrEP-Related Perceived Barriers}

When examining PrEP-related barriers among YMSM with prior PrEP knowledge $(N=410)$, over a third $(36.4 \%)$ agreed that they would not take PrEP due to side effect concerns $(\mathrm{M}=2.30, \mathrm{SD}=.93)$. Close to half $(48 \%)$ of the PrEP-aware sample reported knowing how to access PrEP if they wanted it $(\mathrm{M}=2.47, \mathrm{SD}=1.06)$. YMSM were most likely to agree or strongly agree $(61.2 \%)$ that they could not afford to take $\operatorname{PrEP}(\mathrm{M}=2.78, \mathrm{SD}=.92)$.

In a multivariate model (see Table 3 ) examining concerns about PrEP side effects (F12,392=2.09; $p<.05)$, Black $(\mathrm{b}=.57, \mathrm{se}=.17 ; p<.01)$ and Latino $(\mathrm{b}=.31, \mathrm{SE}=.13 ; p<.05)$ YMSM were more likely than their White counterparts to state that they would avoid using PrEP due to side effect concerns. No other racial/ethnic differences were noted. We 
found no association by age, education, residential instability, incarceration, insurance coverage, URAI in prior two months, or prior STIs, respectively.

We found no association between YMSM's belief that they would know how to get PrEP if they wanted it and the independent variables in our multivariate model (F12,394=1.32; n.s.). When asked whether they would not be able to afford PrEP $(\mathrm{F} 12,390=4.80 ; p<.001)$, we found that Asian/Pacific Islanders $(b=-.71, \mathrm{SE}=.22 ; p<.01)$ were less likely than Whites to note that cost would be a barrier. We found no other racial/ethnic differences regarding PrEP affordability. YMSM who reported having insurance $(b=-$ $.53, \mathrm{SE}=.13 ; p<.001)$ were less likely than those who were uninsured to perceive that they would not be able to afford PrEP YMSM who reported having a STI in the past $(b=33$, $\mathrm{SE}=.11 ; p<.01)$ were more likely to state that they would not be able to afford PrEP Although a marginal association, older YMSM were more likely to state that they would not be able to afford PrEP $(b=.05, \mathrm{SE}=.03 ; p<.10)$. We noted no association between YMSM's affordability belief and age, education, history of incarceration, or prior PrEP awareness, respectively.

\section{DISCUSSION}

In light of its potential to help curtail new HIV infections among YMSM when combined with other forms of prevention such as behavioral interventions, it is vital that we acknowledge and address YMSM's PrEP-related perceived barriers as part of psychosocial interventions aiming to increase PrEP access. Given the limited data on YMSM's awareness and perceived barriers regarding PrEP, we examined whether these constructs were associated with YMSM's sociodemographic characteristics, structural vulnerabilities, and/or HIV risk correlates using a large sample of single YMSM in the United States.

Less than a third of the sample (27\%) reported prior awareness of PrEP. PrEP awareness in our sample is just slightly higher than estimates reported in studies published prior to the release of the iPrEX results (e.g., 20\% in Voetsch et al. [25], 16\% in Liu et al. [26], 19\% in Mimiaga et al. [3], and $36 \%$ in Mehta et al. [27]). These findings suggest that greater attention should be placed on increasing PrEP awareness through public health campaigns. In multivariate analyses, PrEP awareness was higher among those with a history of STIs. Considering that most YMSM reported having heard about PrEP through the media and/or Internet, it is plausible that YMSM with a history of STIs may have an increased perceived susceptibility to HIV/STIs, keeping themselves informed about new advances in HIV prevention and treatment. PrEP awareness campaigns may also benefit by targeting YMSM with lower educational attainment and who are not insured; however, these campaigns must be mindful to be sensitive to literacy concerns. Furthermore, given that most YMSM agreed that PrEP-related affordability was a perceived barrier, care should also be taken to avoid increasing PrEP awareness if YMSM will be unable to afford PrEP.

Concern about PrEP cost was higher among uninsured participants and those with a prior STI history. Although those with insurance were less likely to perceive cost as a barrier, we did not ascertain whether YMSM with different kinds of medical insurance (e.g., public vs private) would be associated with different cost perceptions and/or whether

Table 3. Multivariate Regression of the Relationship between YMSM's PrEP-Related Perceived Barriers and their Sociodemographic Characteristics, Structural Vulnerabilities, and Sexual Risk Correlates

\begin{tabular}{|c|c|c|c|c|c|c|}
\hline & \multicolumn{3}{|c|}{$\begin{array}{l}\text { I would Not Take PrEP Drugs } \\
\text { Because I am Concerned About their Side Effects }\end{array}$} & \multicolumn{3}{|c|}{ I Could Not Afford PrEP Drugs } \\
\hline & B & $\mathbf{S E}$ & Sig. & b & SE & Sig. \\
\hline (Constant) & 1.75 & .56 & .002 & 2.38 & .53 & .001 \\
\hline Age & .02 & .03 & .453 & .05 & .03 & .085 \\
\hline Latino & .31 & .13 & .018 & -.06 & .12 & .617 \\
\hline Asian/Pacific Islander & .31 & .23 & .184 & -.71 & .22 & .002 \\
\hline Multiracial & .40 & .25 & .115 & .21 & .24 & .381 \\
\hline Other & -.37 & .48 & .444 & -.67 & .45 & .141 \\
\hline Ever sent to jail/prison & .16 & .21 & .449 & .23 & .20 & .246 \\
\hline Covered by insurance & .22 & .14 & .116 & -.53 & .13 & .001 \\
\hline URAI in past 2 months & -.16 & .10 & .112 & -.05 & .10 & .585 \\
\hline Prior STI/HIV & -.02 & .11 & .846 & .33 & .11 & .002 \\
\hline
\end{tabular}

Notes: Regression analyses are computed only for YMSM who reported prior PrEP awareness (N=410). Outcomes are scored on a 4-point scale (1=Strongly Disagree; 4=Strongly Agree). 
their insurance would actually cover PrEP. The affordability concerns among YMSM with prior STI history, a group often identified as a high-risk population, is also problematic, as they have been identified as a population that may benefit from PrEP yet may understand the medical costs associated with treatment. Taken together, these cost-related findings are particularly troublesome given ongoing national discussions about rising medical costs and diminishing federal funds for HIV/AIDS prevention and care. Given that the efficacy of PrEP in the iPrEx trial was highest among participants who had greater adherence to the product, the long-term success of PrEP will require YMSM to have access to a combination of biomedical and comprehensive HIV primary prevention services (e.g., access to behavioral counseling, free condoms, treatment for STIs, regular HIV testing, and frequent medical evaluation), as these measures have been shown to increase adherence [1]. At present, however, it remains unclear whether comprehensive services will be available at low or no cost to YMSM, or whether our medical system's infrastructure will be able to sustain these costs. Future research examining the cost-benefit analyses of PrEP rollout and sustainability of services promoting optimal PrEP adherence recommendations is warranted, particularly given that PrEP are not $100 \%$ effective.

Beyond cost concerns, a third of PrEP-aware YMSM perceived that side effects would dissuade them from using PrEP. Although YMSM's beliefs regarding PrEP side effects were less salient in our sample than in Mustanski et al.'s study [17], the difference may be attributable to item wording. In their study, they provided explicit examples of side effects (e.g., dizziness, diarrhea, vomiting, headaches, rash, gas or skin discoloration), whereas we did not. Consequently, participants in our study may not have fully considered different side effects that may emerge as a result of PrEP. Item wording notwithstanding, we noted that African American and Latino YMSM, respectively, were more likely than White counterparts to note that they would not take PrEP if they experienced side effects. This is particularly problematic given that African American and Latino YMSM communities would benefit the most from PrEP from an epidemiological standpoint. We offer two possible interpretations for these findings. First, given public health's history of giving medications/administering treatments to vulnerable racial/ethnic minority populations (e.g., Tuskegee) without fully disclosing all of the risks, it is hardly surprising that there is heightened concern about potential side effects. Second, researchers have also indicated that Black and Latino individuals have both delayed and poorer quality of care $[28,29]$; thus, it is possible that Black and Latino YMSM perceive that side effects would be more difficult to manage and treat than other YMSM in our sample. Support for this interpretation comes from Smith et al.'s qualitative study [16] with African American young adults, where one MSM participant noted that "...[the side effects are] worse than...[the symptoms] you have. Then, to make it worse, they will say it could cause death. That's what's sticking out in my head" (p. 414). Taken together, these findings underscore the importance of addressing PrEP-related side effects, considering the role of medical mistrust in African American and Latino YMSM's hesitance to use PrEP, and creating a comprehensive and accessible medical system where YMSM taking PrEP may receive care for any side effects.

We found no association between YMSM's perceived barrier regarding accessibility and the study variables, or between our structural vulnerability indicators (e.g., residential instability and incarceration) and PrEP awareness and barriers, respectively. The absence of findings may be attributable to our inability to measure structural vulnerabilities beyond the individual level. Although accounting for structural characteristics at the individual level is routine in HIV/AIDS behavioral research, the influence of these social processes may require us to measure them at the community level. Consistent with prior research documenting a relationship between communitylevel characteristics and condom use among young adults [30-32], it is possible that the relationship between structural conditions and PrEP-related barriers (e.g., accessibility) is dependent on institutional (e.g., ease of navigating medical systems) and community-level indicators (e.g., number of AIDS Service Organizations, HIV/AIDS stigma, or concentrated economic disadvantage in a geographic area). Although unavailable in the current study, we encourage researchers to examine the association between communitylevel indicators and PrEP-related perceived barriers in future research. These data may aid in the development of policies that ensure the availability and equity of PrEP across different socioeconomic contexts, may assist in the identification of social contexts where PrEP rollout may have the greatest reach and impact, and/or may help plan community-based intervention strategies that facilitate PrEP rollout.

Although prior research has cautioned that PrEP availability could promote risk disinhibition in behavioral studies, risk disinhibition has not been documented in PrEP trials - particularly with young men. We found no relationship between recent URAI acts and PrEP awareness or perceived barriers, respectively. The absence of a relationship contrasts prior findings by Golub et al. [2] and Mustanski et al. [17], and may be attributable to the fact that we did not ascertain PrEP intentions in our study. Nevertheless, the lack of an association suggests that PrEP awareness - in and of itself - is not promoting greater risk disinhibition among YMSM. Given that only a small number (11 participants) had reported taking PrEP prior to having sex, we were also unable to examine if these participants were more likely to engage in URAI. Assuming that PrEP becomes more readily available and adopted by YMSM, research examining the likelihood of risk disinhibition will be warranted.

While our study provides important insight into PrEPrelated awareness and perceived barriers among YMSM, there are several limitations that must be noted. First, the majority of our sample identified as gay or bisexual, such that our findings may not be generalizable to YMSM who do not claim these identities. Second, although our study includes a national sample of YMSM, it does not necessarily reflect a representative sample of YMSM in the United States as we used a convenience sample of men recruited via the Internet. Therefore, our data may not be generalizable. Third, our survey focused on single YMSM. As a result, it is possible that YMSM in relationships, particularly those with 
sexual agreements, in serodiscordant relationships, and/or in open relationships, may be more aware of PrEP and report different PrEP-related concerns. Fourth, self-report and social desirability bias may have influenced how participants answered survey questions. Finally, it is possible that YMSM's perceived barriers will change as PrEP initiatives are developed and other challenges associated with longterm PrEP use are identified. As a result, it will be imperative that we remain vigilant and address structural and psychosocial obstacles as they are identified.

These limitations notwithstanding, our recently completed study contributes to the literature by acknowledging that PrEP awareness remains low (e.g., less than a third of YMSM) and that PrEP-related concerns seem to be higher among YMSM who might benefit most from this biomedical technology, including youth who are African American or Latino and those without health insurance. Taken together, our findings underscore the importance of considering YMSM's sociodemographic characteristics and structural vulnerabilities in the development of interventions focused on PrEP, as well as questioning the long-term sustainability of PrEP as a large-scale prevention strategy as our public health and medical infrastructures face ongoing financial strains.

\section{CONFLICT OF INTEREST}

None to disclose.

\section{ACKNOWLEDGMENTS}

This research was supported by an NIH Career Development Award (K01-MH087242) to Dr. Bauermeister.

\section{PATIENT CONSENT}

Declared none.

\section{HUMAN/ANIMAL RIGHTS}

Declared none.

\section{REFERENCES}

[1] Grant RM, Lama JR, Anderson PL, et al. Preexposure Chemoprophylaxis for HIV Prevention in Men Who Have Sex with Men. N Engl J Med 2010; 363(27): 2587-99.

[2] Golub SA, Kowalczyk W, Weinberger C, Parsons JT. Preexposure Prophylaxis and Predicted Condom Use Among High-Risk Men Who Have Sex With Men. J Acquir Immune Defic Syndr 2010; 54(5): 548-55.

[3] Mimiaga MJ, Case P, Johnson CV, Safren SA, Mayer KH. Preexposure Antiretroviral Prophylaxis Attitudes in High-Risk Boston Area Men Who Report Having Sex With Men: Limited Knowledge and Experience but Potential for Increased Utilization After Education. J Acquir Immune Defic Syndr 2009; 50(1): 77-83.

[4] Roberts ET, Matthews DD. HIV and chemoprophylaxis, the importance of considering social structures alongside biomedical and behavioral intervention. Soc Sci Med 2012;75(9):1555-61.

[5] Hall HI, Song R, Rhodes P, et al. Estimation of HIV incidence in the United States. JAMA 2008; 300(5): 520-9.

[6] Eaton DK, Kann L, Kinchen S, et al. Youth risk behavior surveillance - United States, 2011. Morbidity and mortality weekly report. Surveillance Summaries 2012; 61(4): 1-162.
[7] CDC. HIV/AIDS and young men who have sex with men. Atlanta: U.S. Department of Health and Human Services, Centers for Disease Control and Prevention; 2009.

[8] Millett GA, Peterson JL, Flores SA, et al. Comparisons of disparities and risks of HIV infection in black and other men who have sex with men in Canada, UK, and USA: a meta-analysis. Lancet 2012; 380(9839): 341-8.

[9] Diaz R, Ayala G. Social Discrimination and Health: The Case of Latino Gay Men and HIV Risk. New York: The Policy Institute of the National Gay and Lesbian Task Force; 2001.

[10] Kipke MD, Weiss G, Wong CF. Residential status as a risk factor for drug use and HIV risk among young men who have sex with men. AIDS Behav 2007; 11(6 Suppl): S56-69.

[11] Millett GA, Flores SA, Peterson JL, Bakeman R. Explaining disparities in HIV infection among black and white men who have sex with men: a meta-analysis of HIV risk behaviors. AIDS 2007; 21(15): 2083-91.

[12] Oster AM, Wiegand RE, Sionean C, et al. Understanding disparities in HIV infection between Black and White MSM in the United States. AIDS 2011; 25(8): 1103-12.

[13] Pace JE, Siberry GK, Hazra R, Kapogiannis BG. Preexposure Prophylaxis for Adolescents and Young Adults at Risk for HIV Infection: Is an Ounce of Prevention Worth a Pound of Cure? Clin Infect Dis 2013; 56(8): 1149-55.

[14] Carpenter CJ. A meta-analysis of the effectiveness of health belief model variables in predicting behavior. Health Commun 2010; 25(8): 661-9.

[15] Rotheram-Borus MJ, Swendeman D, Flannery D, Rice E, Adamson DM, Ingram B. Common factors in effective HIV prevention programs. AIDS Behav 2009;13(3): 399-408.

[16] Smith DK, Toledo L, Smith DJ, Adams MA, Rothenberg R. Attitudes and program preferences of African-American urban young adults about pre-exposure prophylaxis (PrEP). AIDS Educ Prev 2012; 24(5): 408-21.

[17] Mustanski B, Johnson AK, Garofalo R, Ryan D, Birkett M. Perceived Likelihood of Using HIV Pre-exposure Prophylaxis Medications Among Young Men Who Have Sex with Men. AIDS Behav 2013; 17(6): 2173-9.

[18] Millet GA, Peterson JL, Wolitski RJ, Stall R, Millett GA. Greater Risk for HIV Infection of Black Men Who have Sex with Men: A Critical Literature Review. AJPH 2006; 96(6): 1007-19.

[19] Flores SA, Bakeman R, Millett GA, Peterson JL. HIV risk among bisexually and homosexually active racially diverse young men. Sex Transm Dis 2009; 36(5): 325-9.

[20] Liu AY, Vittinghoff E, Sellmeyer DE, et al. Bone mineral density in HIV-negative men participating in a tenofovir pre-exposure prophylaxis randomized clinical trial in San Francisco. PloS One 2011; 6(8): e23688.

[21] Rudy BJ, Kapogiannis BG, Lally MA, et al. Youth-Specific Considerations in the Development of Preexposure Prophylaxis, Microbicide, and Vaccine Research Trials. J Acquir Immune Defic Syndr 2010; 54: S31-S42.

[22] Bauermeister JA, Pingel E, Zimmerman M, Couper M, CarballoDieguez A, Strecher VJ. Data Quality in HIV/AIDS Web-Based Surveys: Handling Invalid and Suspicious Data. Field Methods 2012; 24(3): 272-91.

[23] Couper MP. Designing Effective Web Surveys. New York, NY: Cambridge University Press; 2008.

[24] Bauermeister JA. Romantic ideation, partner-seeking, and HIV risk among young gay and bisexual men. Arch Sex Behav 2012; 41(2): 431-40.

[25] Voetsch AC, Heffelfinger JD, Begley EB, Jafa-Bhushan K, Sullivan PS. Knowledge and Use of Preexposure and Postexposure Prophylaxis Among Attendees of Minority Gay Pride Events, 2005 Through 2006. J Acquir Immune Defic Syndr 2007; 46(3): 378-80.

[26] Liu AY, Kittredge P V, Vittinghoff E, et al. Limited Knowledge and Use of HIV Post- and Pre-Exposure Prophylaxis Among Gay and Bisexual Men. J Acquir Immune Defic Syndr 2008; 47(2): 241-7.

[27] Mehta SA, Silvera R, Bernstein K, Holzman RS, Aberg JA, Daskalakis DC. Awareness of post-exposure HIV prophylaxis in high-risk men who have sex with men in New York City. Sex Transm Infect 2011; 87(4): 344-8.

[28] Flores SA, Mansergh G, Marks G, Guzman R, Colfax G. Gay identity-related factors and sexual risk among men who have sex with men in San Francisco. AIDS Educ Prev 2009; 21(2): 91-103. 
[29] Parrish DD, Kent CK. Access to care issues for African American communities: implications for STD disparities. Sex Transm Dis 2008; 35(12 Suppl): S19-22.

[30] Bauermeister JA, Zimmerman MA, Caldwell CH. Neighborhood Disadvantage and Changes in Condom Use among African American Adolescents. J Urban Health 2011; 88(1): 66-83.
[31] Frye V, Koblin B, Chin J, et al. Neighborhood-Level Correlates of Consistent Condom Use among Men Who have Sex with Men: A Multi-Level Analysis. AIDS Behav 2010; 14(4): 974-85.

[32] Johns MM, Bauermeister JA, Zimmerman MA. Individual and Neighborhood Correlates of HIV testing among african american youth transitioning from adolescence into young adulthood. AIDS Educ Prev 2010; 22(6): 509-22. 